J. Austral. Math. Soc. 19 (Series A) (1975), 431-436.

\title{
ORDERING FINITE GROUPS BY INVOLVEMENT
}

\author{
M. D. ATKINSON
}

(Received 27 October 1972)

Communicated by G. E. Wall

\section{Introduction}

In the study of locally finite varieties of groups it has often been illuminating to consider when a group $A$ is a factor (i.e. quotient of a subgroup) of a group $B$. We write $A \preccurlyeq B$ to express this and say that $A$ is involved in $B$. It follows from elementary isomorphism theorems that the relation $\preccurlyeq$ is a partial order on any set of finite groups. The conjecture that we consider in this paper (and to which we only give the beginning of an answer) is the following:

CONJECTURE. The relation of involvement is a partial well-order on the set of finite groups of a Cross variety. In other words, in a Cross variety there does not exist an infinite set of finite groups no one of which is a factor of another.

As evidence that this is an interesting question we mention two results. The first can be proved by easy arguments about partially ordered sets and the second can be proved using the techniques of Kovács and Newman as described in Chapter 5 of Neumann (1967).

Proposition 1. If $\mathfrak{X}$ is any factor closed set of finite groups the following conditions are equivalent:

i) $\mathfrak{X}$ is partially well-ordered by involvement

ii) The factor closed subsets of $\mathfrak{X}$ satisfy the minimum condition

iii) There are at most countably many factor closed subsets of $\mathfrak{X}$.

Proposition 2. Let $\mathfrak{B}$ be a locally finite variety whose finite groups are not partially well-ordered by involvement. Then, if $S$ is any non-abelian finite simple group and $\mathfrak{U}=\operatorname{var}(S), \mathfrak{U} \mathfrak{B}$ has uncountably many subvarieties.

(This can be compared with a result of Olshanskii (1970): if the finite monolithic groups of a locally finite variety $\mathfrak{B}$ are not partially well-ordered by involvement then, for any prime $p$ not dividing the exponent of $\mathfrak{B}, \mathfrak{A}_{p} \mathfrak{B}$ contains uncountably many subvarieties. It was partly by using this result that Olshanskii obtained the first known set of uncountably many varieties.) 
The conjecture is obviously true for abelian Cross varieties and there is at least one other case where its truth can be established fairly easily (see Cossey (1966), my thanks to the referee for supplying this reference):

PROPOSITION 3. If $p$ and $q$ are distinct primes then the set of finite groups of $\mathfrak{A}_{p} \mathfrak{H}_{q}$ is partially well-ordered by involvement.

In the case of $\mathfrak{A}_{p} \mathfrak{A}_{q}$ the relation of involvement is the same as the subgroup relation and this simplifies the argument. Since the same thing is true of any set of finite $E A$-groups (groups which have elementary abelian Sylow $p$-subgroups for all primes $p$ ) one might hope that the conjecture should not be too intractable for Cross varieties of $E A$-groups. We shall consider the conjecture for the variety $\mathfrak{T}_{p}$ (of all groups of exponent $p$ and nilpotency class at most 2) which is one of the smallest cases where not all the groups are $E A$-groups. In this variety the relation of involvement is not the same as the subgroup relation - indeed, P. M. Neumann (unpublished) has shown that there exists an infinite set of finite groups in $\mathfrak{I}_{p}$ no one of which is a subgroup of another. However, the main result of this paper states that this cannot happen with the involvement relation.

THEOREM. The set of finite groups of $\mathfrak{I}_{p}$ is partially well-ordered by involvement.

We shall not give the complete proof of this theorem (full details can be found in Atkinson (1970). Instead we give an outline of the proof and the details of some of the crucial steps. Those parts which are omitted require arguments which are very similar in spirit (but different in detail) to the arguments involved in the study of alternating trilinear forms in Atkinson (1973).

This work was carried out while I was supported by an S.R.C. scholarship. It was supervised by Dr. P. M. Neumann to whom I am indebted for many helpful suggestions.

\section{Proper factor closed subsets of $\mathfrak{I}_{p}$}

Let $\mathfrak{I}_{p}^{*}$ denote the set of finite groups of $\mathfrak{I}_{p}$. To prove the theorem we have to consider an arbitrary infinite subset $\mathfrak{X}$ of $\mathfrak{I}_{p}^{*}$ and show that for some $G$, $H \in \mathfrak{X}, G \nsubseteq H$ we have $G \preccurlyeq H$. If, in the notation of Neumann (1967), $Q S \mathfrak{X}=\mathfrak{I}_{p}^{*}$ this conclusion is easily obtained. For let $G \in \mathfrak{X}$ and let $K$ be any group in $\mathfrak{I}_{p}^{*}$ with $G \preccurlyeq K$ and $G \nsubseteq K$; then, as $Q S \mathfrak{X}=\mathfrak{I}_{p}^{*}$, there is a group $H \in \mathfrak{X}$ such that $K \preccurlyeq H$ and thus $G \preccurlyeq H$. In this section we shall describe a useful consequence of the contrary case $Q S \mathfrak{X} \subset \mathfrak{I}_{p}^{*}$.

In order to state this consequence we define some special factor closed subsets of $\mathfrak{I}_{p}^{*}$.For any two non-negative integers $a, b$ let $\mathfrak{B}(a, b)=\left\{G \in \mathfrak{I}_{p}^{*} \mid G\right.$ has a subgroup $H$ such that $[G: H] \leqq p^{a}$ and $\left.\left|H^{\prime}\right| \leqq p^{b}\right\}$. An obvious calculation establishes that $\mathfrak{B}(a, b)$ is indeed factor closed. The main result of this section is the following lemma. 
LEMMA 2.1 Any proper factor closed subset $\mathfrak{X}$ of $\mathfrak{T}_{p}^{*}$ is contained in some $\mathfrak{B}(a, b)$.

Proof. Let $E$ denote the non-abelian group of exponent $p$ and order $p^{3} . E$ is the unique generating critical group of $\mathfrak{I}_{p}$ and so every group of $\mathfrak{I}_{p}^{*}$ is a factor of some direct power $E^{n}$. Since $\mathfrak{X} \neq \mathfrak{I}_{p}^{*}$ there exists an integer $m$ such that, for all $G \in \mathfrak{X}, E^{m+1}$ not $\preccurlyeq G$. We shall complete the proof of the lemma by showing that $E^{m+1}$ not $\preccurlyeq G$ implies $G \in \mathfrak{B}(a, b)$ where $a$ and $b$ depend only on $m$.

For the remainder of the proof $G$ will denote a group in $\mathfrak{I}_{p}^{*}$ such that $E^{m+1}$ not $\preccurlyeq G$. Let $n$ be the greatest integer for which $E^{n} \preccurlyeq G$; so that $n \leqq m$. Then there exist subgroups $H$ and $K$ of $G$ such that $K \triangleleft H$ and $H / K \cong E^{n}$. Among all pairs $(H, K)$ satisfying this condition we choose one with $|H|$ minimal; it follows then that $K \leqq H^{\prime}$. Since $H / K \cong E^{n}$ there exist elements $a_{1}, \cdots, a_{2 n} \in H$ such that the $n$ elements $c_{i}=\left[a_{2 i-1}, a_{2 i}\right]=\left[a_{2 i}, a_{2 i-1}\right]^{-1}$ are independent modulo $K$ and all other commutators $\left[a_{i}, a_{j}\right] \in K$. Clearly, $H=\left\langle a_{1}, \cdots, a_{2 n}\right\rangle$, $H^{\prime}=\left\langle c_{1}, \cdots, c_{n}, K\right\rangle$ and $\left|H^{\prime}\right| \leqq p^{4 n^{2}}$.

Choose a subgroup $T$ such that $T \times H^{\prime}=G^{\prime}$ and let $C$ be the subgroup $C=\{x \in G \mid[x, H] \leqq T\}$. Then $C$ is the preimage of $C_{G / T}(H T / T)$ and so, as $\left|(G / T)^{\prime}\right|=\left|H^{\prime}\right|,[G: C] \leqq p^{4 n^{2} \cdot 2 n}=p^{8 n^{3}}$.

Now let $z$ and $t$ be arbitrary elements of $C$. We shall show that $[z, t] \in[z, H]$ $[t, H] H^{\prime}$. Suppose that this is not so. Consider any relation of the form

$$
\prod_{i=1}^{n} c_{i}^{\alpha_{i}}[z, t]^{\alpha_{n+1}} \in K[z, H][t, H]
$$

Since $\prod_{i=1}^{n} c_{l}^{\alpha_{i}}$ and $K$ are contained in $H^{\prime}$ we have that $[z, t]^{\alpha_{n+1}} \in[z, H][t, H] H^{\prime}$ and, from our supposition, $\alpha_{n+1} \equiv 0(\bmod p)$. Thus $\left(^{*}\right)$ implies that $\prod_{i=1}^{n} c_{i}^{\alpha_{t}}$ $\in K[z, H][t, H]$ and so we may write $\prod_{i=1}^{n} c_{i}^{\alpha_{t}}=k s$ where $k \in K \leqq H^{\prime}$ and $s \in[z, H][t, H]$. Consequently

$$
\prod_{i=1}^{n} c_{i}^{\alpha_{i}} k^{-1}=s \in H^{\prime} \cap T=1
$$

and thus $\prod_{i=1}^{n} c_{i}^{\alpha_{i}} \in K$ and from our assumption on $c_{1}, \cdots, c_{n}$ we have $\alpha_{i} \equiv 0$ $(\bmod p)$ for $i=1, \cdots, n$.

Thus, putting $c_{n+1}=[z, t]$, we have that $c_{1}, \cdots, c_{n+1}$ are independent modulo $K[z, H][t, H]$ and it follows that

$$
\langle H, z, t\rangle K / K[z, H][t, H] \cong E^{n+1} .
$$

This contradiction shows that $[z, t] \in[z, H][t, H] H^{\prime}$.

It follows that, for some $u, v \in H$ and $w \in H^{\prime}$, we have $[z, t]=[z, u][t, v] w$. Since $\left[u^{-1}, v\right] \in H^{\prime}$ this implies that $\left[z v, t u^{-1}\right] \in H^{\prime}$ and so $z v \in C\left[t u^{-1} \bmod H^{\prime}\right)$, the preimage of the centraliser of $t u^{-1} H^{\prime}$ in $G / H^{\prime}$. Thus 


$$
z \in\left(C\left(t u^{-1} \bmod H^{\prime}\right) H\right) \cap C \subseteq \bigcup_{u \in H}\left(C\left(t u^{-1} \bmod H^{\prime}\right) H\right) \cap C \subseteq C .
$$

But $z$ is an arbitrary element of $C$ and so it follows that

$$
C=\bigcup_{u \in H}\left(C\left(t u^{-1} \bmod H^{\prime}\right) H\right) \cap C .
$$

Now $C\left(t u^{-1} \bmod H^{\prime}\right)$ depends only on the coset $u^{-1} H^{\prime}$ and not on $u$ itself. Since $\left|H / H^{\prime}\right|=p^{2 n} C$ is the union of $p^{2 n}$ subgroups and so one of these subgroups has index not exceeding $p^{2 n}$. Thus, for some $w \in H,\left[C:\left(C\left(t w^{-1} \bmod H^{\prime}\right) H\right)\right.$ $\cap C] \leqq p^{2 n}$. From this it follows that $\left[C: C\left(t w^{-1} \bmod H^{\prime}\right) \cap C\right] \leqq p^{4 n}$. Hence, given any element $t \in C$, we can find $w \in H$ such that

$$
\left[C: C\left(t w^{-1} \bmod H^{\prime}\right) \cap C\right] \leqq p^{4 n} .
$$

Consider an arbitrary subgroup of $C$ generated by $2 n+1$ elements $t_{1}, \cdots, t_{2 n+1}$ which are independent modulo $C^{\prime} H^{\prime}$. As we have just shown there exist elements $w_{1}, \cdots, w_{2 n+1}$ of $H$ such that

$$
\left[C: C\left(t_{i} w_{i}^{-1} \bmod H^{\prime}\right) \cap C\right] \leqq p^{4 n} \quad \text { for } i=1, \cdots, 2 n+1 \text {. }
$$

Now $H$ can be generated by $2 n$ elements and so there exist integers $\alpha_{1}, \cdots, \alpha_{2 n+1}$ not all zero modulo $p$ such that $\prod_{i=1}^{2 n+1} w_{i}^{\alpha_{i}} \in H^{\prime}$. Then

$$
x=\prod_{i=1}^{2 n+1} t_{i}^{\alpha_{i}} \equiv \prod_{i=1}^{2 n+1} t_{i}^{\alpha_{i}} \prod_{i=1}^{2 n+1} w_{i}^{-\alpha_{i}} \equiv \prod_{i=1}^{2 n+1}\left(t_{i} w_{i}^{-1}\right)^{\alpha_{i}} \bmod G^{\prime}
$$

and so $x$ satisfies $\left[C: C\left(x \bmod H^{\prime}\right) \cap C\right] \leqq p^{(2 n+1) 4 n}$ and $x \in X-C^{\prime}$.

Each element $y$ of $C$ induces by commutation a linear mapping $C / C^{\prime} H^{\prime} \rightarrow$ $C^{\prime} H^{\prime} / H^{\prime}$ with rank $\beta(y)$ say. The function $\beta(y)$ satisfies $\beta(y+z) \leqq \beta(y)+\beta(z)$, $\beta(\lambda y)=\beta(y)$ for any non-zero scalar $\lambda$ and $\beta(0)=0$. Also, we have shown that given any $2 n+1$ dimensional subspace $X$ of $C / C^{\prime} H^{\prime}$ there is a non-zero element $x$ of $X$ such that $\beta(x) \leqq(2 n+1) 4 n$. By Lemma 2.7 of Atkinson (1973) there is a subspace $D / C^{\prime} H^{\prime}$ of $C / C^{\prime} H^{\prime}$ such that $[C: D] \leqq p^{2 n}$ and, for all $d \in D / C^{\prime} H^{\prime}$, $\beta(d) \leqq 8 n(2 n+1)^{2}$. A theorem of P. M. Neumann (1970) now gives that $\left|D^{\prime} H^{\prime}\right| H^{\prime} \mid \leqq p^{64 n^{2}(2 n+1)^{4}}$. However, the index of $D$ in $G$ is

and also

$$
[G: C][C: D] \leqq p^{8 n^{3}+2 n} \leqq p^{8 m^{3}+2 m}
$$

$$
\left|D^{\prime}\right| \leqq p^{64 n^{2}(2 n+1)^{4}} \leqq p^{64 m^{2}(2 m+1)^{4}}
$$

and so the proof is completed.

\section{Groups with bounded derived subgroups}

In the remainder of the proof of the theorem we have to consider an infinite set of groups $\mathfrak{X}$ whose factor closure is not the whole of $\mathfrak{I}_{p}^{*}$. We know that, 
for some $a$ and $b, \mathfrak{X} \subseteq \mathfrak{B}(a, b)$. In the case $b=0$ arguments very like those used in Atkinson (1973) in the study of alternating trilinear forms can be used and so these details will be omitted. Instead, in this section, we concentrate on the case $x=0$ and explain the main ideas. In the general case a group $G \in \mathfrak{B}(a, b)$ can be regarded as a mixture of a group in $\mathfrak{B}(a, 0)$ and a group in $\mathfrak{B}(0, b)$ because it has i subgroup $H$ such that $H \in \mathfrak{B}(0, b)$ and $G / H^{\prime} \in \mathfrak{B}(a, 0)$. Because of this the general zase can be attacked with the results from the two special cases and here again we omit the details.

A useful property of $\mathfrak{I}_{p}^{*}$ which was important in section 2 was that $\mathfrak{I}_{p}^{*}$ is he factor closure of the set $\left\{E^{n}\right\}_{n=1}^{\infty}$ and these groups are easily described. We low consider a corresponding set of groups which may be used in the study of $\mathcal{B}(0, b)$. We define $E_{n}$ to be the extraspecial group of exponent $p$ and order $p^{2 n+1}$ so that $E_{1}=E$ ) and we have the following result:

LEMMA 3.1 Every group in $B(0, b)$ is a subgroup of $E_{n}^{b}$ for some $n$.

The proof of this is not unlike the proof of Theorem 2.2 of Atkinson (1973) Ind so will be omitted. In the remainder of this section we investigate conditions which guarantee that $E_{n}^{b}$ is a subgroup of the group $G$ in $\mathfrak{B}(0, b)$. This requires inother definition. For any non-abelian group $G \in \mathfrak{I}_{p}^{*}$ we define $m(G)$, the minimal ank of $G$, as follows: with every maximal subgroup $M$ of $G^{\prime}$ there is an associated Iternating bilinear form induced by commutation in $G / M ; m(G)$ is the minimum sver all maximum subgroups $M$ of $G^{\prime}$ of the ranks of these forms. Two simple acts about the minimal rank of $G$ which concern a subgroup $H$ of index $p^{n}$ are

a) if $m(G)>2 n$ then $H^{\prime}=G^{\prime}$

b) $m(H) \geqq m(G)-2 n$.

LEMMA 3.2 Let $G$ be a group in $\mathfrak{I}_{p}^{*}$ which satisfies the following two conlitions

i) $\left|G^{\prime}\right|=p^{b}, b \geqq 1$

ii) $m(G) \geqq 4 b^{2}$

Then $E_{1}^{b}$ is a subgroup of $G$.

Proof. Since $G$ is not abelian $E_{1}$ is a subgroup of $G$. Suppose inductively hat $E_{1}^{i} \leqq G, i<b$. Let $A=C\left(E_{1}^{b}\right)$ so that $[G: A]<p^{2 b^{2}}$. By a) above $A^{\prime}=G^{\prime}$. ince $\left(E_{1}\right)^{\prime}\left\langle A^{\prime}\right.$ there exist $c, d \in A$ such that $[c, d] \notin\left(E_{1}^{i}\right)^{\prime}$. Clearly $\left\langle E_{1}^{i}, c, d\right\rangle$ $=E_{1}^{i+1}$ and this completes the proof.

LEMMA 3.3 Let $G$ be a group in $\mathfrak{I}_{p}^{*}$ which satisfies the following two con!itions

i) $\left|G^{\prime}\right|=p^{b}, b \geqq 1$

ii) $m(G) \geqq 4 b^{2} N n$ where $N=|G L(b, p)|+1$

Then $G$ has a subgroup isomorphic to $E_{n}^{b}$. 
Proof. Let $M=N n$. We first construct subgroups $D_{1}, \cdots, D_{M}$ of $G$ such that $D_{i} \cong E_{1}^{b}$ and, for $i \neq j,\left[D_{i}, D_{j}\right]=1$, The existence of $D_{1}$ is guaranteed by Lemma 3.2. Suppose inductively that $D_{1}, \cdots, D_{i}, i<M$, have been constructed and satisfy all the required conditions. Let $A=C\left(D_{1} \cdots D_{i}\right)$ so that $[G: A] \leqq p^{2 i b^{2}}$. By a) above $A^{\prime}=G^{\prime}$ and by b) $m(A) \geqq 4 b^{2} N n-4 i b^{2} \geqq 4 b^{2}$. Applying Lemma 3.2 to $A$ we obtain a subgroup $D_{i+1} \leqq A, D_{l+1} \cong E_{1}^{b}$ and for all $j \leqq i$, $\left[D_{j}, D_{i+1}\right]=1$. This completes the inductive step.

Since, for each $i, D_{i} \cong E_{1}^{b}$ we may choose generators $c_{i}^{(1)}, d_{i}^{(1)}, \cdots, c_{i}^{(b)}, d_{i}^{(b)}$ of $D_{i}$ such that

$$
\begin{array}{ll}
{\left[c_{i}^{(r)}, d_{i}^{(s)}\right]=e_{i}^{(r)^{d} r s}} & 1 \leqq r, s \leqq b \\
{\left[c_{i}^{(r)}, c_{i}^{(s)}\right]=\left[d_{i}^{(r)}, d_{i}^{(s)}\right]=1} & 1 \leqq r, s \leqq b
\end{array}
$$

and $e_{i}^{(1)}, \cdots, e_{i}^{(b)}$ are generators for $G^{\prime}$.

Now there are less than $N$ choices for a generating set of $b$ elements for $G^{\prime}$ and hence among the $N n$ generating sets $\left\{c_{i}^{(1)}, \cdots, c_{i}^{(b)}\right\}_{i=1}^{M}$ for $G^{\prime}$ one must occur at least $n$ times. By relabelling $D_{1}, \cdots, D_{M}$ and by relabelling the generating sets for each $D_{i}$ we may assume that $e_{i}^{(j)}=e_{k}^{(j)}$ for $j=1, \cdots, b$ and $i, k=1, \cdots n$. If we let $F_{j}=\left\langle c_{j}^{(j)}, d_{i}^{(j)} \mid 1 \leqq i \leqq n\right\rangle$ then we have $F_{j} \cong E_{n}$ and since the $F_{j}$ clearly generate their direct product the lemma is proved.

\section{References}

M. D. Atkinson (1970), D. Phil. thesis, (Oxford, 1970)

M. D. Atkinson (1973), 'Alternating trilinear forms and groups of exponent 6', J. Austral Math. Soc. 16, 111-128.

Hanna Neumann (1967), Varieties of groups. (Berlin-Heidelberg-New York, Springer Verlag, 1967).

P. M. Neumann (1970), 'An improved bound for BFC p-groups', J. Austral. Math. Soc. 11, 19-27.

A. Ju. Olshanskii (1970), 'On the problem of a finite basis for the identities of a group'. (Russian) Izv. Akad. Nauk SSSR 34, 376-384.

John Cossey (1966), On varieties of A-groups, Ph. D. thesis, (Australian National University 1966).

Department of Computing Mathematics

University College

Cardiff 